\title{
A MULTINATIONAL ECONOMIC EVALUATION OF rhDNase IN THE TREATMENT OF CYSTIC FIBROSIS
}

\author{
Joseph Menzin \\ Gerry Oster \\ Policy Analysis Inc.
}

Linda Davies

Michael F. Drummond

University of York

Wolfgang Greiner

Universitat Hannover

Carlo Lucioni

Istituto Economia Sanitaria

Jean-Louis Merot

Benefit International

Franco Rossi

Istituto Economia Sanitaria

J. Graf v.d. Schulenburg

Universitat Hannover

Eric Souêtre

Benefit International

\begin{abstract}
Economic evaluations of pharmaceuticals are increasingly being conducted in conjunction with randomized phase III clinical trials to meet the demand for pharmacoeconomic data when new products are launched. While the need for such data is often global, the trials in which relevant information may be collected are often conducted in only one or a limited number of countries. A critical issue is how data from pivotal clinical trials in one setting can serve as the basis for pharmacoeconomic evaluations in others. We address this issue and report on four economic evaluations that we undertook in conjunction with a recent U.S. phase III clinical trial of recombinant human deoxyribonuclease (rhDNase), which is used to improve pulmonary function in patients with cystic fibrosis (CF). The objective of these evaluations was to estimate the potential impact of rhDNase therapy in France, Germany, Italy, and the United Kingdom on the direct costs of medical care for the treatment of respiratory tract infections (RTIs) in patients with CF. Analyses of economic impact were undertaken
\end{abstract}


both with and without adjustment for differences in practice patterns between the United States and the countries of interest. Our findings suggest that rhDNase therapy may reduce the cost of RTI-related care by between US $\$ 600$ and US $\$ 1,100$ over a 24-week period; the cost of rhDNase is not included in these figures, as a price was unavailable when our analyses were undertaken. Despite methodologic challenges, economic evaluations that meet the information needs of decision makers in diverse countries can nonetheless be undertaken in conjunction with phase III clinical trials.

Economic evaluations of pharmaceuticals are increasingly being conducted in conjunction with randomized phase III clinical trials. Such evaluations often are perceived to be more credible than studies conducted on a retrospective basis (1). They also are often the only source of cost-effectiveness data when new products are launched. A growing number of countries require submission of health economic data to obtain approval for reimbursement or to negotiate a favorable price with government authorities $(2 ; 4)$.

In trial-based evaluations, economic effects are estimated using information on resource utilization collected prospectively during the conduct of a formal clinical experiment. Outcomes are specified in advance and assessed through direct observation over a predetermined period of follow up. Retrospective economic analyses, in contrast, often rely heavily on the use of modeling to project expected outcomes and costs. The numerous assumptions (and often data sources) employed in such analyses may leave them open to criticism.

Many of the methodologic issues that are encountered in undertaking trial-based economic evaluations have been explored in detail $(6 ; 7)$. However, little attention has been paid to the questions of whether - and how - pivotal clinical trials conducted in one setting can serve as the basis for economic evaluations in others (5). This problem arises because phase III clinical trials are typically conducted in only one or a limited number of countries, while the need for data on economic impact is increasingly global in nature.

In this paper, we address this issue and report on findings from four European economic evaluations that we undertook in conjunction with a recent U.S. phase III clinical trial of recombinant human deoxyribonuclease (rhDNase) (PULMOZYME, Genentech, Inc., South San Francisco, CA). rhDNase was recently approved for use in the United States and several European countries to improve pulmonary function in persons with cystic fibrosis.

\section{METHODS}

\section{Cystic Fibrosis and rhDNase}

Cystic fibrosis (CF) is one of the most common fatal inherited disorders (8). Although multisystemic, it is characterized by the accumulation in the lungs of thick mucus, which limits pulmonary function and often leads to recurrent infections. More than one third of CF patients in the United States are hospitalized each year, and one in five are hospitalized two or more times annually (3).

The highly viscous nature of the mucus in the lungs of CF patients is due in large part to a high concentration of deoxyribonucleic acid (DNA). The human gene

We would like to thank Felix Streichenberg, Ph.D., and Todd Rich, M.D., M.B.A., for their significant contribution to this project, and to Meredith Regan, B.A., for her help in preparing the databases used in the study. 
for deoxyribonuclease (DNase), which reduces the viscosity of purulent sputum, was recently cloned and has been used to produce rhDNase (14). In early clinical trials, aerosolized rhDNase was reported to improve pulmonary function $\left(\mathrm{FEV}_{1}\right)$ by $10-$ $15 \%$ in patients with CF $(11 ; 12)$.

To further document the safety and efficacy of rhDNase, a phase III clinical trial was recently undertaken (9). A total of $968 \mathrm{CF}$ patients 5 years of age and older were randomized to rhDNase $2.5 \mathrm{mg}$ twice daily (BID) $(n=321), 2.5 \mathrm{mg}$ once daily (QD) $(n=322)$, or vehicle $(n=325)$, and treated for 24 weeks. The average age of trial patients was about 19 years, and one half were female. Study therapy was administered via nebulizer on an outpatient basis. Primary outcomes of interest included change in pulmonary function, which was assessed biweekly, and the incidence over 24 weeks of respiratory tract infections (RTIs) requiring parenteral antibiotic therapy.

While the clinical benefits of rhDNase were anticipated to be significant, a reduced incidence of respiratory tract infection (RTI) also was anticipated to have important economic implications, leading to savings in the associated costs of treating RTIs in patients with CF. For this reason, data were collected prospectively during the phase III clinical trial on a number of measures of health care resource utilization, and formal health economic evaluations were planned.

For each trial participant, information was collected prospectively on the numbers of hospital admissions and associated inpatient days, and the numbers of days of oral and intravenous antibiotic therapy. The reason for administering each antibiotic was also recorded on study case report forms (i.e., "treatment of respiratory tract infection," "prophylaxis," and "other") and used to determine whether treatment was RTI-related. The design of the trial as well its principal findings are reported elsewhere (9).

\section{Design of European Health Economic Assessments}

Overview. An international project team was assembled to explore the effects of rhDNase therapy on RTI-related health care utilization and costs. The team consisted of experts in clinical economics from four European countries; the project was coordinated by health economists from the United States (G. O. and J. M.). It was agreed at the outset that all investigators had the right to publish findings from these studies.

Objective and Methodologic Approach. The objective of this project was to estimate the expected impact of rhDNase therapy on RTI-related health care utilization and costs among CF patients in France, Germany, Italy, and the United Kingdom. To facilitate the comparison of findings across countries, it was agreed at the outset that a similar methodology would be used in all countries. Attention was focused on estimating the potential effect of rhDNase therapy on the costs of RTI-related care over 24 weeks in each country of interest. Since rhDNase was not a marketed product at the time these analyses were undertaken (i.e., 1992-93) and its price therefore was unknown, the cost of the study drug was not included in our estimates of economic impact.

To estimate the effect of rhDNase therapy on the costs of RTI-related care, measures of physical resource use (i.e., the numbers of hospital admissions, inpatient days, and days of oral and intravenous antibiotic therapy) were compared between patients who received rhDNase versus placebo in the U.S. phase III trial. Hospitalizations were designated as RTI-related if an antibiotic was given parenterally and the 
investigator indicated that the reason for therapy was "treatment of respiratory tract infection."

Differences in RTI-related resource use were then evaluated using local (i.e., country-specific) estimates of unit costs. Alternative estimates of economic impact also were derived after adjustment for differences in practice patterns (i.e., between the countries of interest and the United States). To facilitate comparisons of findings across countries, we converted costs expressed in European currencies to U.S. dollars using purchasing power parities (10).

Estimation of Unit Costs. In each country of interest, institutions were identified for the purpose of collecting information on the daily costs of inpatient and outpatient treatment for CF patients with RTIs. The centers chosen and the precise costing methodologies that were employed differed by country.

In Germany, where hospitals are reimbursed on a per-diem basis, the lowest per-diem cost among three specialized CF centers (university clinics in Hannover, Essen, and Frankfurt) (DM 510) was used in the analysis. Since drug costs are included in this per diem, and the use of antibiotics was documented in the phase III clinical trial and costed in the economic evaluations, this amount was reduced by $7 \%$, or the proportion of the per diem typically spent on medication. In France a per-diem cost was similarly calculated using the lowest reimbursed amount (FF 2,991) from two CF centers (university clinics in Paris and Lyon).

In Italy accounting data for individual departments in a single CF center (a university clinic in Milano) were aggregated to estimate a mean daily cost of care (ItL 647,000). The components of cost included personnel, drugs other than antibiotics, diagnostic procedures, hotel (e.g., catering, laundry and linen, cleaning), equipment and maintenance, and overhead. In the United Kingdom the median estimate (UKf 200) from three CF centers (London, Northern Ireland, and Northwest England) was used to calculate daily costs in a manner similar to that used for Italy.

Antibiotics prescribed for treatment of RTIs also were costed using local prices. If an antibiotic that was prescribed in the U.S. phase III trial was unavailable locally, the lowest price of a commonly used alternative was used instead (if one could be identified), or it was excluded from the cost analysis.

Assessment of Differences in Practice Patterns. The reduction in the risk of RTI among patients who received rhDNase in the phase III trial was believed to be generalizable to other settings. It was anticipated, however, that patterns of treatment for CF patients with RTI might differ. The use of resource utilization data from the U.S. phase III clinical trial could therefore lead to misestimation of the expected economic impact of rhDNase therapy on the costs of RTI-related care in the countries of interest. The "practice pattern" parameters that we believed most likely to vary across countries were the likelihood of hospitalization (i.e, for $\mathrm{CF}$ patients with RTIs) and the associated mean length of stay in hospital.

For this reason, estimates of the values of these two parameters were obtained in each of the countries of interest. In two of the countries (Germany and the United Kingdom), discussions with CF experts indicated that the frequency of hospitalization was comparable to that observed in the U.S. phase III clinical trial (i.e., about $80 \%$ ). However, it was learned that patients who are hospitalized in the United States often are treated instead on an outpatient basis in France and Italy. In France the expected rate of hospitalization (approximately $60 \%$ ) was determined through discussions with clinical experts, while in Italy this rate $(56 \%)$ was estimated based on interviews with 


\section{Menzin et al.}

clinicians as well as a review of case records for patients presenting to a CF center (University of Milano) with RTIs.

To determine the average length of hospital stay for RTI in France, Germany, and Italy, case notes for approximately $\mathbf{3 0}$ hospitalized patients were reviewed in each of these countries. The mean number of days in the hospital for treatment of RTI was estimated to be 11.4 in France, 14.2 in Italy, and 14.4 in Germany; the average among patients in the placebo group of the U.S. phase III trial was 12.3 days. In the United Kingdom clinical experts at four CF centers indicated that the mean length of hospitalization was also approximately 12 days. These differences were not believed to be large enough to warrant adjustment.

Analyses. Analyses of data focused on documenting differences in resource utilization between the rhDNase QD and placebo groups in the phase III clinical trial and estimating the economic implications of these differences in each of the countries of interest. The rhDNase BID group was not included in these analyses, because this dose is only recommended for limited use in Europe (i.e., in some persons over 21 years of age).

A Wilcoxon rank-sum test was used to examine the significance of differences in continuous measures of resource use (e.g., days in hospital for treatment of RTI), while a chi-square test was used for discrete measures (e.g., incidence of hospitalization). Estimates of treatment costs were not subjected to formal statistical testing.

Practice-adjusted analyses were undertaken in Italy and France to account for differences (i.e., between these countries and the United States) in the likelihood of hospitalization for patients with an RTI. The expected impact of rhDNase therapy on the costs of RTI-related care was estimated by multiplying the difference in the mean number of RTIs per patient between treatment groups (i.e., rhDNase QD versus placebo) by an estimate of the average cost of treating an RTI. In the practice-adjusted analysis, we calculated the latter as a weighted average of local inpatient and outpatient treatment costs, where the weight corresponded to the likelihood of treatment in each of these settings.

There also was concern that the centers in which cost data were collected might not be representative of all $\mathrm{CF}$ centers in the countries of interest. Sensitivity analyses were therefore undertaken using alternative estimates of the daily costs of inpatient treatment. In Germany the average per-diem cost for all hospitals (DM 350) was used as a lower bound, while the cost estimate from the highest cost CF center (DM 790) was used as an upper bound. A high value only (FF 7,512) was used in the sensitivity analysis in France, since the base case estimate was thought to be conservative. In Italy national estimates of the daily cost of hospital care in pediatric departments were used to determine low (ItL 547,000) and high (ItL 774,000) values. In the United Kingdom the lowest and highest estimates of daily inpatient costs from three CF centers were used (UK£ 145 and UK£ 347, respectively).

\section{RESULTS}

\section{Clinical Outcomes}

Among patients randomized to the rhDNase QD group in the phase III clinical trial, $\mathrm{FEV}_{1}$ was $5.8 \%$ higher on average during follow-up than at baseline, while there was no change in this measure in the placebo group; this difference was significant 
Table 1. Health Care Utilization Over 24 Weeks for Patients Randomized to rhDNase Once Daily or Placebo in the U.S. Phase III Clinical Trial, by Reason

\begin{tabular}{lcc}
\hline & \multicolumn{2}{c}{ Treatment Group } \\
\cline { 2 - 3 } Health Care Utilization, by Reason & Placebo & rhDNase QD \\
\hline Number of patients & 325 & 322 \\
Hospital admissions (mean) & 0.56 & $0.41^{+}$ \\
$\quad$ Respiratory tract infection & 0.62 & $0.50^{+}$ \\
$\quad$ Any reason & & \\
Inpatients days (mean) & 6.4 & $4.9^{+}$ \\
$\quad$ Respiratory tract infection & 6.9 & $5.6^{+}$ \\
$\quad$ Any reason & 6.2 & $4.8^{+}$ \\
Days of inpatient intravenous antibiotic therapy (mean) & 6.5 & 5.2 \\
$\quad$ Respiratory tract infection & & \\
$\quad$ Any reason & 0.55 & 0.59 \\
Days of inpatient oral antibiotic therapy (mean) & 1.04 & 1.00 \\
$\quad$ Respiratory tract infection & & \\
$\quad$ Any reason & 4.4 & $2.9^{+}$ \\
Days of outpatient intravenous antibiotic therapy (mean) & 6.1 & $3.1^{++}$ \\
$\quad$ Respiratory tract infection & & \\
$\quad$ Any reason & 25.2 & 23.5 \\
Days of outpatient oral antibiotic therapy (mean) & 63.7 & 62.1 \\
$\quad$ Respiratory tract infection & & \\
Any reason & & \\
\hline
\end{tabular}

$+\mathrm{p}<.05$ by Wilcoxon rank sum test.

${ }^{++} \mathrm{p}<.01$ by Wilcoxon rank sum test.

$(p<.001)$. The incidence of RTI over 24 weeks also was significantly lower in the rhDNase group ( $34 \%$ versus $43 \%$ for placebo; $p<.05)(9)$.

\section{Economic Outcomes}

Differences in resource utilization between patients receiving rhDNase QD and placebo in the phase III clinical trial are shown in Table 1 . The mean number of hospitalizations for treatment of an RTI was significantly lower in the rhDNase QD group $(p<.05)$, as was the corresponding mean number of inpatient days (4.9 versus $6.4 ; p<.05)$. The mean numbers of days of RTI-related inpatient and outpatient intravenous antibiotic therapy also were lower among patients randomized to rhDNase.

Estimates of the economic impact of rhDNase therapy on the costs of RTI-related care over 24 weeks, excluding the cost of the study drug, are shown in Table 2. Reductions in the costs of RTI-related care (excluding the cost of rhDNase) are estimated to range from about UK£ 434 (US $\$ 700$ ) in the United Kingdom to approximately FF 7,011 (US $\$ 1,100$ ) in France. Lower costs of inpatient treatment represent the largest component of these savings. Using the lowest estimates of daily inpatient costs, savings range from UKf 300 (US $\$ 480$ ) to DM 1,784 (US \$850); the corresponding range based on the highest daily costs is ItL $1,500,000$ (US $\$ 1,000$ ) to FF 13,872 (US $\$ 2,100$ ).

In the practice-adjusted analyses, rhDNase was found to reduce treatment costs by approximately ItL 900,000 (US $\$ 600$ ) and FF 5,640 (US $\$ 860$ ); these estimates represent about $75 \%$ of the amounts estimated without adjustment, reflecting lower assumed rates of hospitalization than those observed in the phase III trial. Details of these calculations may be found in the Appendix. 
Table 2. Difference in Mean Cost of RTI-related Care (Placebo Minus rhDNase) Excluding Cost of Study Medication Over 24 Weeks in Local Currencies and U.S. Dollars, by Country

\begin{tabular}{|c|c|c|c|c|}
\hline \multirow[b]{2}{*}{ Component of Cost } & \multicolumn{4}{|c|}{ Country } \\
\hline & France & Germany & Italy & U.K. \\
\hline & \multicolumn{4}{|c|}{ Costs in Local Currency } \\
\hline Inpatient Care & & & & \\
\hline Days in hospital & FF 4,540 & DM 711 & ItL 982,000 & $£ 300$ \\
\hline Antibiotic therapy & 806 & -1 & 122,000 & 50 \\
\hline Outpatient Care & 1,665 & -1 & 181,000 & 84 \\
\hline \multirow[t]{2}{*}{ Total } & 7,011 & 1,970 & $1,285,000$ & 434 \\
\hline & \multicolumn{4}{|c|}{ Costs in US\$ ${ }^{2}$} \\
\hline \multicolumn{5}{|l|}{ Inpatient Care } \\
\hline Days in hospital & 693 & 337 & 660 & 477 \\
\hline Antibiotic therapy & 123 & -1 & 82 & 79 \\
\hline Outpatient Care & 254 & -1 & 122 & 134 \\
\hline Total & 1070 & 934 & 864 & 690 \\
\hline
\end{tabular}

\section{DISCUSSION}

In a recent U.S. randomized phase III placebo-controlled clinical trial, rhDNase was found to improve pulmonary function and reduce the incidence of RTI in persons with $\mathrm{CF}$. To document the economic implications of these clinical findings, a formal health economic assessment was undertaken in conjunction with this trial. An international project team was assembled to estimate the likely economic impact of rhDNase therapy on the costs of RTI-related care in France, Germany, Italy, and the United Kingdom. The findings from these evaluations suggest that rhDNase would be likely to reduce the costs of RTI-related care by between US $\$ 600$ and $\$ 1,100$ over a period of 24 weeks.

Because rhDNase was not a marketed product at the time these investigations were undertaken, its cost was not included in our analyses. rhDNase has since been approved for use in a number of countries. For example, the price to the public in Switzerland is SFR 52 per ampule (13) (about US \$24) (10), or approximately $\$ 4,000$ over 24 weeks, assuming once daily administration; the cost of therapy may vary somewhat between countries. Our findings therefore suggest that while rhDNase would reduce the costs of RTI-related care, it would nonetheless be expected to lead to higher health care costs among persons with CF.

While rhDNase may increase costs of care for $\mathrm{CF}$, its use may nonetheless be cost-effective. Not all of the relevant costs of RTI-related care were captured in our study. For example, patients with RTI may require additional physician visits as well as respiratory therapy; neither were documented in this study. We also focused only on those costs that would be incurred by hospitals and/or national health authorities, or so-called direct costs. Indirect costs incurred by patients or their families (e.g., time lost from work) were not documented. By not including these costs, we may have understated the economic benefits of a reduced incidence of RTI. In addition, use of rhDNase may have other benefits, including improvement in patient well-being. 
In the phase III clinical trial, patients in the rhDNase groups experienced less dyspnea and reported improved overall well-being and fewer CF-related symptoms (9). However, the value that patients place on these outcomes, as well as being free of infection and remaining out of hospital, is not known. Finally, the relatively short period of follow-up used in this trial precluded examining whether rhDNase can prolong patient survival. Further data from clinical trials are therefore needed before questions of cost-effectiveness can be thoroughly investigated.

The approach that we adopted relied heavily on the use of data collected during a randomized clinical trial. We believe that the random assignment of patients to treatment and the collection of data prospectively over a defined period of follow-up (i.e., 24 weeks) provided internally valid estimates of the effect of rhDNase on levels of health care utilization. However, we recognized that important differences may exist between treatment practices in the United States (where the phase III trial was conducted) and other countries of interest. We therefore collected data in each country to ascertain whether practice patterns varied. As a result of this effort, key differences in treatment patterns were identified (i.e., more frequent use of outpatient parenteral antibiotics in France and Italy), and estimates of the projected economic impact of rhDNase were adjusted accordingly.

Little attention has been paid to how clinical trials conducted in one setting can serve as the basis for economic evaluations in others. In this paper, we have described findings from an economic assessment of rhDNase that was undertaken in Europe based on data on resource use collected during a U.S. phase III clinical trial. We have also highlighted a methodologic approach that overcomes many of the difficulties inherent in model-based studies, yet allows sufficient flexibility to account for important differences in practice patterns. We believe that our study can serve as a model for other similar investigations.

\section{APPENDIX: CALCULATION OF PRACTICE-ADJUSTED COSTS OF RTI-RELATED CARE}

To calculate the expected economic impact of rhDNase on a practice-adjusted basis (PACOST), the mean numbers of RTIs per patient (RTI) in the rhDNase and placebo groups of the U.S. clinical trial were multiplied by the corresponding expected cost of treating an RTI, and the difference in treatment costs was calculated. The expected cost of an RTI was calculated as a weighted average of the costs of inpatient (INPTCOST) and outpatient (OUTPTCOST) treatment; the weight corresponded to the likelihood of being treated in France and Italy in each of these settings (PHOSP, 1 - PHOSP). The formula for calculating practice-adjusted costs of RTI-related care in each treatment group can be expressed as:

$$
\text { PACOST }=\text { RTI * }[\text { PHOSP * INPTCOST }+(1-\text { PHOSP }) * \text { OUTPTCOST }]
$$

To estimate INPTCOST, measures of resource use from the U.S. phase III trial (e.g., number of days in hospital, number of days of inpatient intravenous antibiotic therapy) were expressed in terms of averages per RTI episode, and local estimates of corresponding costs were applied. The costs of these individual components of treatment were then summed. OUTPTCOST was calculated in a similar manner. Table Al describes our findings from the practice-adjusted analyses. 
Menzin et al.

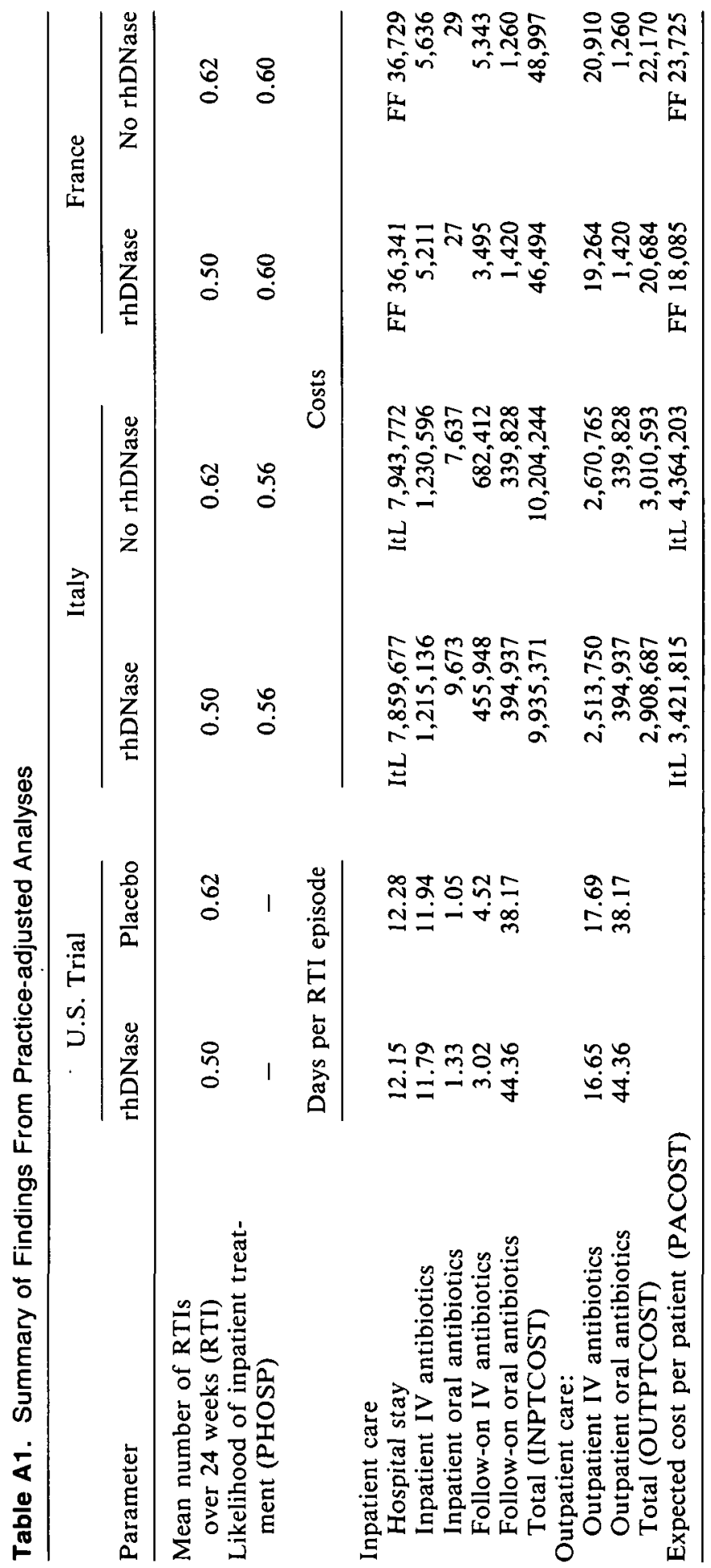




\section{REFERENCES}

1. Anders, G. Doubts are cast on cost studies by drug makers. Wall Street Journal, June 28, 1994, section B, 1 .

2. Commonwealth of Australia. Guidelines for the submission of data to the Pharmaceutical Benefits Advisory Committee (PBAC) including economic analysis. Canberra, 1992.

3. Cystic Fibrosis Foundation Patient Registry. Annual data report, 1991, Cystic Fibrosis Foundation, Bethesda, MD: 1992.

4. Drummond, M. F. Cost-effectiveness guidelines for reimbursement of pharmaceuticals: Is economic evaluation ready for its enhanced status? Health Economics, 1992, 1, 85-92.

5. Drummond, M. F., Bloom, B. S., Carrin, G., et al. Issues in the cross-national assessment of health technology. International Journal of Technology Assessment in Health Care, 1992, 8, 671-82.

6. Drummond, M. F., \& Davies, L. Economic analysis alongside clinical trials: Revisiting the methodological issues. International Journal of Technology Assessment in Health Care, 1991, 7, 561-73.

7. Drummond, M. F., \& Stoddart, G. L. Economic analysis and clinical trials. Controlled Clinical Trials, 1984, 5, 115-28.

8. FitzSimmons, S. C. The changing epidemiology of cystic fibrosis. Journal of Pediatrics, 1993, 122, 1-9.

9. Fuchs, H. J., Borowitz, D. J., Christiansen, D. H., et al. Effect of aerosolized recombinant human DNase on exacerbations of respiratory symptoms and on pulmonary function in patients with cystic fibrosis. New England Journal of Medicine, 1994, 331, 637-42.

10. Organization for Economic Cooperation and Development. National accounts, vol. I: Main aggregates, 1960-92. Paris: OECD. 1994, 155

11. Ramsey, B. W., Astley, S. J., Aitkey, M. L., et al. Efficacy and safety of short-term administration of aerosolized recombinant human deoxyribonuclease in patients with cystic fibrosis. American Review of Respiratory Disease, 1993, 148, 145-51.

12. Ranasinha, C., Assoufi, B., Shak, S., et al. Efficacy and safety of short-term administration of aerosolized recombinant DNase $I$ in adults with stable stage cystic fibrosis. Lancet 1993, 342, 199-202.

13. Reglementation, Monatsliste, abcd Spezialitäten Nachtrag zum Katalog. Geneva. 1994, $15.6,1$.

14. Shak, S., Capon, D. J., Hellmiss, R., et al. Recombinant human DNase I reduces the viscosity of cystic fibrosis sputum. Proceedings of the National Academy of Science USA, $1990,87,9188-92$. 